\title{
Escala Crenças Parentais sobre Competência Emocional em Crianças (COMPE): Propriedades Psicométricas
}

\author{
Deise Maria Leal Fernandes Mendes*, 1 \\ Orcid.org/0000-0003-3487-7284 \\ Pedro P. Pires ${ }^{2}$ \\ Orcid.org/0000-0001-8831-9056
}

Ana Carolina Monnerat Fioravanti ${ }^{3}$

Orcid.org/0000-0003-2340-4043

\author{
${ }^{1}$ Universidade do Estado do Rio de Janeiro, Rio de Janeiro, RJ, Brasil \\ ${ }^{2}$ Universidade Federal do Rio de Janeiro, Rio de Janeiro, RJ, Brasil \\ ${ }^{3}$ Universidade Federal Fluminense, Rio das Ostras, RJ, Brasil
}

\section{Resumo}

Crenças parentais sobre competências emocionais das crianças representam o que pais pensam e acreditam a respeito de como a criança se desenvolve em termos de habilidades ligadas a emoções e de quais capacidades principais nesse domínio deve desenvolver. A competência emocional é um componente crítico e impactante para o desenvolvimento pleno e saudável. O objetivo desse estudo foi o de apresentar, brevemente, as etapas de construção da escala COMPE e verificar os parâmetros psicométricos preliminares como dimensionalidade e confiabilidade dos itens. Para atingir a forma final da escala, foram adotados procedimentos de Validade de Conteúdo para a constituição da versão inicial e de Teoria de Resposta ao Item para a análise das propriedades psicométricas dos itens. Como resultado, o presente artigo apresenta uma escala com um total de 30 itens capazes de compor uma medida para as crenças parentais mencionadas, com três facetas teóricas indicativas de competências emocionais ligadas a: (a) expressão emocional, (b) compreensão emocional e (c) regulação emocional, que são agregadas sob um escore único. Com base nas propriedades psicométricas verificadas, considera-se que a COMPE passa a ser uma opção de instrumento a ser utilizado com população brasileira em pesquisas envolvendo crenças parentais e emoções.

Palavras-chave: Desenvolvimento emocional, crenças (não religiosas), escalas, COMPE.

\section{Scale Parental Beliefs about Emotional Competence in Children (COMPE): Psychometric Properties}

\footnotetext{
Abstract

Parental beliefs regarding the emotional skills of children represent what parents think and believe about how the child develops in terms of emotion-related skills and which key abilities should be developed

* Endereço para correspondência: Universidade do Estado do Rio de Janeiro, Rua São Francisco Xavier, 524, $10^{\circ}$ andar, Maracanã, Rio de Janeiro, RJ, Brasil 20550-900. E-mail: deisefmendes@gmail.com, ana@fioravantiana. org e ppires85@gmail.com

Apoio financeiro: Fundação de Amparo à Pesquisa do Estado do Rio de Janeiro (FAPERJ) e Conselho Nacional de Desenvolvimento Científico e Tecnológico (CNPq).
} 
in this area. Emotional skill is a high impact component critical for full and healthy development. The aim of this study was to present, briefly, the construction steps of the COMPE scale and to verify the preliminary psychometric parameters, such as the dimensionality and reliability of the items. In order to reach the final form of the scale, Content Validity procedures were used for the constitution of the initial version and Item Response Theory (IRT) for the analysis of the psychometric properties of the items. As a result, the present article presents a scale with a total of 30 items capable of composing a measure for the parental beliefs mentioned, with three theoretical facets indicative of emotional skills linked to: (a) emotional expression, (b) emotional comprehension and (c) emotional regulation, which are aggregated into a single score. Based on the psychometric properties verified, it is considered that the COMPE can become an instrument option to be used with the Brazilian population in research involving parental beliefs and emotions.

Keywords: Emotional development, beliefs (nonreligious), scaling, COMPE.

\section{Escala Creencias de los Padres sobre la Competencia Emocional en los Niños (COMPE): Propiedades Psicométricas}

\section{Resumen}

Creencias parentales sobre las competencias emocionales de los niños representan lo que los padres piensan y creen acerca de cómo el niño se desarrolla en términos de habilidades vinculadas a emociones y de qué capacidades principales en ese dominio debe desarrollar. La competencia emocional es un componente crítico y impactante para el desarrollo pleno y saludable. El objetivo de este estudio fue presentar brevemente las etapas de construcción de escala COMPE y comprobar los parámetros psicométricos preliminares como dimensionalidad y confiabilidad de los ítems. Para conseguir la forma final de la escala, los procedimientos de Validez de Contenido se adoptaron para la creación de la versión inicial, y de Teoría de Respuesta para analizar las propiedades psicométricas de los ítems. Como resultado, este documento presenta una escala con un total de 30 ítems capaces de componer una medida de las creencias parentales mencionadas, con tres facetas teóricas indicativas de competencias emocionales relacionadas con: (a) expresión emocional, (b) comprensión emocional y (c) regulación emocional, que se agregan en una sola puntuación. Basado en las propiedades psicométricas verificadas, la COMPE se convierte en una opción de instrumento a ser utilizado con población brasileña, en investigaciones que implican emociones y creencias parentales.

Palabras clave: Desarrollo emocional, creencias (no religiosas), escala, COMPE.

Interconexões entre as crenças dos pais e suas práticas parentais constituem evidência e são discutidas na literatura (Harkness \& Super, 2010; Sheffield-Morris, Silk, Steinberg, Myers, \& Robinson, 2007). Tais crenças podem ser transmitidas diretamente aos filhos através de mecanismos de comunicação como narrativas e instruções, afetando os esquemas das crianças sobre suas identidades pessoais e sobre o mundo em geral (Bohanek et al., 2009; Fivush, 2008). Assim, crenças parentais relativas a competências emocionais das crianças parecem ter impacto no desenvolvimento emocional das mesmas por serem guias para o comportamento dos pais, e/ou refletirem inclinações comportamentais dos mesmos, sendo transmitidas aos filhos de modo a infundirem neles entendimentos sobre como lidar com as emoções (Parker et al., 2012).

Ademais, crenças parentais sobre emoção além de associadas a competências essenciais do desenvolvimento emocional das crianças, como a regulação emocional, compreensão de emoções e expressão emocional, são sensíveis ao contexto cultural em que a criança se desenvolve (Denham, 2007; Trommsdorff \& Cole, 2011). Conhece-las constitui uma via relevante para a 
ampliação do conhecimento acerca da parentalidade e do desenvolvimento emocional infantil, expandindo possibilidades de atuação na busca de um desenvolvimento pleno e saudável (Lozada, Halberstadt, Craig, Dennis, \& Dunsmore, 2016; Trommsdorff \& Cole, 2011). No entanto, pouco se conhece sobre crenças parentais relacionadas às emoções, em especial em pais brasileiros. Como agravante, não se dispunha de instrumentos para avaliação do fenômeno nesta população.

A relevância das competências ligadas à emoção para que se tenha uma vida saudável, biológica e socialmente, vem sendo reconhecida e sublinhada por psicólogos e neurocientistas nas últimas décadas (Damásio, 2009; Denham, Ferrier, Howarth, Herndon, \& Bassett, 2016; Finlon et al., 2015; Goleman, 1995; Saarni, 1999). Alguns construtos ligados a esse domínio das habilidades humanas vêm sendo propostos, com ênfases diferenciadas nos aspectos diversos a serem levados em conta, sendo a noção de inteligência emocional, formulada por Goleman (1995) um dos que alcançou grande repercussão dentro e fora da Psicologia.

Definida por esse autor como um conjunto de capacidades que inclui controle de impulsos, automotivação, empatia e competência social em relações interpessoais, algumas iniciativas foram realizadas com o propósito de instrumentalizar a mensuração da inteligência emocional, como a Escala Veiga de Competência Emocional (Mendonça, 2009) e o Emotional Competence Inventory (ECI; Boyatzis \& Sala, 2004). O desenvolvimento dessas capacidades na ontogênese e a visão de pais a este respeito, contudo, não parecem fazer parte das preocupações que nortearam essa formulação teórica e pesquisas associadas.

Partindo de outra perspectiva teórica, Halberstadt et al. (2013) trabalharam a ideia de crenças parentais sobre as emoções das crianças. Segundo os autores, constituem aspectos importantes da socialização parental de emoção e podem estar relacionadas com a saúde mental e o bem-estar das crianças. Assim, desenvolveram um questionário multifacetado para avaliar as crenças dos pais sobre emoções infantis, o $\mathrm{Pa}$ rents' Beliefs about Children's Emotions Ques- tionnaire (PBACE), composto por sete escalas, cobrindo as reações e formas de encarar lidar com as manifestações emocionais dos filhos. Entretanto, talvez o mais conhecido trabalho empírico referente às crenças dos pais a respeito de emoções seja a meta-emoção parental (Gottman, Katz, \& Hooven, 1996). Embora as medidas desenvolvidas de meta-emoção demonstrem pontos fortes psicométricos, o construto em si combina avaliação de crenças e comportamentos, o que torna difícil distinguir o impacto das crenças parentais do que se possa atribuir a comportamentos parentais.

O interesse e a concepção teórica assumida para a construção da escala COMPE, aqui proposta, é diversa das iniciativas mencionadas e se baseia na noção de desenvolvimento da competência emocional, segundo o entendimento de Denham (1998). Conceito multidimensional e complexo, a competência emocional envolve alguns componentes dos quais a autora destaca com ênfase a expressão, a compreensão e a regulação emocionais. Tais componentes funcionam de maneira integrada de acordo com Denham (1998).

As habilidades envolvidas na competência emocional se constituem de acordo com a idade, de modo adequado ao meio cultural e à situação social em questão, envolvendo o reconhecimento em si e nos outros dos processos psicológicos. Assim, a competência emocional é revelada em diversas situações diárias vividas por indivíduos em todas as etapas do ciclo vital e se desenvolve, desde o nascimento, ao longo da vida. Entretanto, é na infância que para Denham (2007) o desenvolvimento da competência emocional ganha espaço importante e requer maior atenção, especialmente entre os três/quatro e cinco anos. Estudos empíricos têm demonstrado que tais habilidades desempenham um papel central nas trajetórias de desenvolvimento de crianças, não apenas com vistas à saúde mental, mas também ao sucesso acadêmico e à competência social, dando suporte ao desenvolvimento cognitivo, à prontidão e ao ajustamento escolar (Curby, Brown, Bassett, \& Denham, 2015; Denham, Bassett, Zinsser, \& Wyatt, 2014; Izard et al., 2001).

Pensando a respeito e investigando o mo- 
mento da ontogênese situado entre os dois e os cinco anos, quando a criança apresenta acentuado progresso no desenvolvimento em várias áreas (fala, pensamento, motora, brincadeira social), Denham (1998) pondera que as crianças são emocionalmente mais sofisticadas do que se poderia pensar. Argumenta que as várias transformações na competência emocional nesse período têm levado psicólogos do desenvolvimento a tentar descrevê-las mais plenamente.

Denham (1998) discute cada uma das três competências centrais ligadas às emoções e considera que a expressão emocional em crianças pequenas inclui: usar gestos para expressar mensagens emocionais não-verbais sobre uma situação social ou relação; demonstrar envolvimento empático com as emoções do outro; exibir emoções de autoconsciência e sociais complexas, como culpa, orgulho, vergonha, em contextos apropriados; e, perceber que alguém pode se sentir de determinado modo "internamente", mas mostrar um comportamento distinto.

Para a compreensão de emoções em crianças pequenas são destacadas por Denham (1998) as habilidades para: discernir seus estados emocionais; discernir os estados emocionais dos outros; e, usar o vocabulário de emoções. No que se refere à regulação emocional, são mencionadas as capacidades para: lidar com emoções aversivas ou angustiantes, ou as situações que as suscitam; lidar com emoções de prazer ou situações que as suscitem; e regular estrategicamente a experiência e expressão emocional em momentos apropriados.

Apesar da relevância das emoções e da parentalidade para os estudos voltados para o desenvolvimento infantil, não foram identificados na revisão da literatura instrumentos nacionais ou internacionais focados em crenças parentais relativas às emoções, avaliando a importância atribuída por mães e pais às habilidades centrais da competência emocional, para o desenvolvimento da criança. Considerando-se a necessidade da avaliação dessas crenças para uma compreensão mais ampla da socialização da emoção de crianças de quatro a cinco anos, sob esse enfoque e a partir de nossa cultura, foi desenvolvida uma escala de Crenças Parentais sobre Com- petência Emocional em Crianças (COMPE).

O objetivo desse estudo foi apresentar brevemente as etapas de construção da escala e verificar os parâmetros psicométricos preliminares como dimensionalidade e confiabilidade dos itens. Parte-se do princípio de que a construção e validação de um instrumento psicológico é de extrema importância para o avanço do conhecimento científico de uma determinada área.

Instrumentos de medida como, por exemplo, as escalas, exigem etapas bem definidas e técnicas rigorosas em sua elaboração (Pasquali, 2010a, 2011), pois pretendem quantificar fenômenos não observáveis ou variáveis latentes, características dos constructos psicológicos. Pasquali (2010a) aponta para três grandes polos ou procedimentos denominados de: polo teórico, polo empírico e polo analítico. Incluem etapas e métodos específicos, que devem ser desenvolvidos em sequência.

Considerando-se que a definição constitutiva é a identificação das dimensões e atributos dos construtos, no presente estudo a etapa da definição constitutiva, foi realizada em função das concepções teóricas formuladas por Denham (1998, 2007). Assim, o objetivo da construção da escala sobre crenças parentais acerca da importância da competência emocional de crianças foi baseado em formulações teóricas, segundo as quais, podem ser focalizados três grandes pilares: a expressão emocional (mudanças potencialmente observáveis na face, voz, corpo, e nível de atividade a partir de algum evento interno ou externo que provoque uma emoção), a compreensão de emoções (capacidade para identificar emoções em si mesmo e nas outras pessoas e saber nomeá-las adequadamente) e a regulação emocional (capacidade para lidar com emoções, sejam as prazerosas ou as angustiantes, e com as situações que as promovem).

Neste estudo, para a definição operacional, foi extraída da literatura uma variedade de conceitos operacionais que representassem expressão emocional (usar gestos, como um abraço, para expressar mensagens emocionais não-verbais sobre uma situação social ou relação, manifestar expressões faciais de emoção como o sorriso), compreensão de emoções (perceber 
que se sente mais tristeza do que raiva quando a professora chama a atenção; saber que o sorriso do pai ao chegar do trabalho deve indicar que está satisfeito), e a regulação emocional (recorrer à mãe quando o irmão pega seu brinquedo ao invés de bater nele).

A partir desses conceitos operacionais, foi produzida uma listagem de categorias que são expressas em tarefas unitárias e específicas, denominadas de itens. De acordo com Pasquali (2010b), a construção dos itens corresponde à etapa de operacionalização do construto e a fonte principal utilizada para o desenvolvimento dessa etapa é a revisão bibliográfica sobre o construto. ${ }^{1}$

Os itens da Escala de Crenças Parentais sobre Competência Emocional em Crianças foram construídos considerando um quantitativo de frases que manifestam expressão emocional, compreensão de emoções e regulação emocional, reunido com base na revisão bibliográfica e na experiência da primeira autora. Optou-se como estrutura do instrumento por uma escala de Likert onde as categorias foram definidas em seis graus associados ao quanto o participante concorda com a afirmativa: nada importante, minimamente importante, pouco importante, razoavelmente importante, muito importante e extremamente importante. Foram construídos 104 itens em função das definições constitutiva e operacional das formulações de Denham (1998, 2007) obedecendo os critérios que, de acordo com Pasquali (1998) são: comportamental, de objetividade ou de desejabilidade, da simplicidade, de clareza, de relevância, de precisão, de variedade, de modalidade, de tipicidade e da credibilidade.

Do total de 104 itens construídos para a Escala de Crenças Parentais sobre Competência Emocional em Crianças, 45 itens foram do componente Expressão emocional, 40 itens do componente Compreensão de emoções e 19 itens do componente Regulação emocional, todos construídos em forma de sentenças a serem qualificadas pelos respondentes. Os itens foram

O pool de itens foi construído a partir dos grupamentos de fenômenos levantados nas temáticas prevalentes. construídos como representantes comportamentais da formulação teórica de Denham (1998). Contudo, torna-se necessário avalia-los mediante a opinião de especialistas na área, conhecidas como juízes, garantindo sua validade (Pasquali, 1998, 2010b). Sendo assim, para a análise semântica dos itens e análise de conteúdo, a $E s$ cala de Crenças Parentais sobre Competência Emocional em Crianças foi submetida à análise por juízes.

Foi solicitado aos juízes, que se posicionassem quanto ao seu nível de concordância nos itens e sua opinião sobre adequação semântica, facilidade de compreensão e adequação da representação comportamental de cada item da escala, podendo descrever suas considerações quanto à construção do item.

O resultado da análise dos juízes culminou na permanência de 73 itens dos 104 iniciais, em função de valores baixos obtidos no coeficiente de validade de conteúdo (CVC) - inferiores a 0,80 . Dos itens da faceta teórica de regulação emocional, cinco foram revisados pelos especialistas. Após as revisões, a escala apresentou CVC igual a 0,99 tanto para adequação, quanto para clareza da redação dos itens, indicando a concordância dos juízes sobre a boa qualidade do conteúdo da escala. Essa versão, composta por 40 itens sobre expressão emocional, 23 a respeito de compreensão emocional e 10 relativos à regulação emocional, foi utilizada para o estudo aqui apresentado, segundo o método descrito a seguir.

\section{Método}

\section{Amostra}

Participaram da pesquisa 81 pais com filhos na idade requerida, dos quais $87,7 \%$ ( $n$ $=71)$ são do sexo feminino e $12,3 \%(n=10)$ do sexo masculino, com idade média de 35,21 anos $(D P=6,48$, com idade mínima de 24 e máxima de 54 anos), em sua maioria provenientes do estado do Rio de Janeiro $(48,1 \%, n=39)$, enquanto metade da amostra foi representada por participantes de um total de nove estados. Quanto à escolaridade, $40 \%(n=32)$ apresentaram pós-graduação completa ou em andamen- 
to, $16 \%(n=13)$ ensino superior completo, $23 \%$ $(n=19)$ ensino superior incompleto e $21 \%(n$ $=17)$ nível educacional inferior ou igual ao ensino médio completo. Considerando-se o estado civil, $85 \%(n=69)$ dos participantes declararam união estável, $5 \%(n=4)$ indicaram a situação de separados(as)/divorciados(as) e 10\% $(n=8)$ a de solteiros(as). Os filhos dos participantes apresentaram idade entre quatro e cinco anos - conforme a faixa etária para uso da escala - com idade média de $M=4,63(D P=0,48)$, sendo crianças no nível da Educação Infantil. Ser (1) brasileiro, (2) residente no país, (3) pai ou mãe, (4) de criança de quatro a cinco anos foram os critérios de inclusão utilizados - e exclusão, quando não contemplados. A coleta de dados foi realizada de junho a agosto de 2016.

\section{Instrumentos}

Questionário sóciodemográfico - participantes reportaram sexo, idade, maior nível de escolaridade atingido, estado civil, renda familiar, profissão, vínculo profissional, localização, data de nascimento do filho que se enquadra na pesquisa e características das pessoas que vivem no domicílio. Parte dos dados será utilizada em pesquisas posteriores.

Escala de Competências Emocionais com o objetivo de medir crenças dos pais acerca de diferentes aspectos da competência emocional, foram selecionadas três facetas teóricas, com um total de 73 itens para a fase de análise dos itens para evidência de validade. A estrutura em faceta representou a teoria sobre a organização das competências emocionais. No entanto, para fins de medida, a escala pontua uma dimensão geral de crença positiva dos pais acerca da importância das competências listadas. Pontuações baixas ou localização em logits inferiores representam participantes com baixo foco ou valorização de competências emocionais em crianças na mesma faixa etária de seus filhos, enquanto o oposto ocorre em pontuações ou localizações superiores. As facetas teóricas consistem em: (1) expressão emocional (40 itens), (2) compreensão emocional (23 itens) e (3) regulação emocional (10 itens).
A escala é apresentada a partir do seguinte texto: "Marque um dos números para dizer o quanto você acha que "é importante para o desenvolvimento da criança que ela...". Respostas poderiam ser assinaladas em uma escala Likert de seis pontos, com descritores variando entre "(1) nada importante", "(2) minimamente importante", "(3) pouco importante", “(4) razoavelmente importante", "(5) muito importante" e "(6) extremamente importante". Em usos no contexto clínico ou de pesquisa, recomenda-se o cálculo da média das respostas ao instrumento. Pesquisas futuras serão conduzidas com o objetivo de produzir as normas de interpretação, assim como percentis de localização com amostra de referência adequada.

\section{Procedimento}

Os participantes responderam a formulários online disponíveis através do Google Docs. A divulgação foi realizada nas redes sociais (Facebook, Linkedin e Google+), assim como por e-mail, com envio de um texto breve de apresentação e o link para o formulário de pesquisa. Antes de responder aos instrumentos, contudo, deveriam primeiramente ler e assentir ao Termo de Consentimento Livre e Esclarecido. Depois da leitura e concordância com a participação na pesquisa, o formulário seguiu na sequência com o questionário sociodemográfico e, por fim, a escala COMPE foi apresentada. Ao completar o formulário, uma opção para envio do formulário preenchido ficava disponível, sucedida por uma frase de agradecimento pela participação. Em estudo-piloto verificou-se que o preenchimento do formulário completo durava de 20 a 30 minutos.

\section{Análise dos Dados}

O software utilizado para as análises foi o R v3.3.1 ( $R$ Core Team, 2016). A análise de dimensionalidade foi conduzida através do pacote nFactors (Raiche \& Magis, 2010). A análise de confiabilidade e dos itens foi conduzida através do pacote mirt (Chalmers, 2012).

A dimensionalidade no pacote nFactors pode ser analisada através de uma pluralidade de 
regras, considerando que retorna a quantidade de fatores através do critério de Kaiser, de autovalores superiores a 1,00. Apresenta também a quantidade de fatores retidos na análise paralela, através do método de coordenadas otimizadas e, por fim, pelo fator de aceleração. Raiche e Magis (2010) recomendam que seja decidido pelo número de fatores retidos ao longo de todos os procedimentos.

A análise de confiabilidade foi conduzida tanto através do coeficiente alfa de Cronbach, normalmente reportado por regra na literatura de psicometria, assim como o coeficiente de confiabilidade empírica (CCE). O CCE é obtido considerando uma estimativa secundária do traço latente e seus erros padrões retornados pelo comando fscores (Chalmers, 2012).

A análise dos itens foi conduzida utilizando o paradigma de Teoria de Resposta ao Item, segundo o Modelo Generalizado de Créditos Parciais (MGCP). Considerando que a presente pesquisa obteve uma amostra inferior ao necessário frente aos desafios de coleta, foi utilizado o método de reamostragem Metropolis-Hastings Robbins-Monro (MHRM), com o intuito de ampliar a robustez das estimativas e análises subsequentes (Chalmers, 2012). Foram utilizados três critérios com o intuito de selecionar itens: (1) teste de ajuste do chi-quadrado para cada item, onde valores não significativos indicam um ajus- te da curva de característica do item aos padrões de resposta observados; e (2) nível de discriminação entre 0,70 e superiores a 1,50 ao mesmo tempo que observando a qualidade da informação dos itens e do teste (Baker, 2001; De Ayala, 2009). Dentre as características dos itens reportadas no modelo MGCP, incluem-se o nível de discriminação e o nível de traço médio apresentado pelos graus de resposta de cada item.

\section{Resultados}

\section{Dimensionalidade}

A investigação inicial da dimensionalidade da escala aponta para um total de 15 dimensões com autovalores superiores a 1,00 , sendo que na análise paralela são apontadas cinco dimensões. Análise das coordenadas otimizadas mantém cinco dimensões são perdidas, entretanto, quatro dimensões na análise do fator de aceleração, restando uma única dimensão que acumula $37 \%$.

Após o processo de remoção dos itens, que será reportado na seção de ajuste dos itens, há uma perda do número de dimensões, sendo que a escala passa a ter sete dimensões detectadas dentro do critério de autovalores: três dimensões para a análise paralela, três dimensões para coordenadas otimizadas e uma dimensão, ao final, para o fator de aceleração, com a retenção de $29 \%$ da variância explicada.

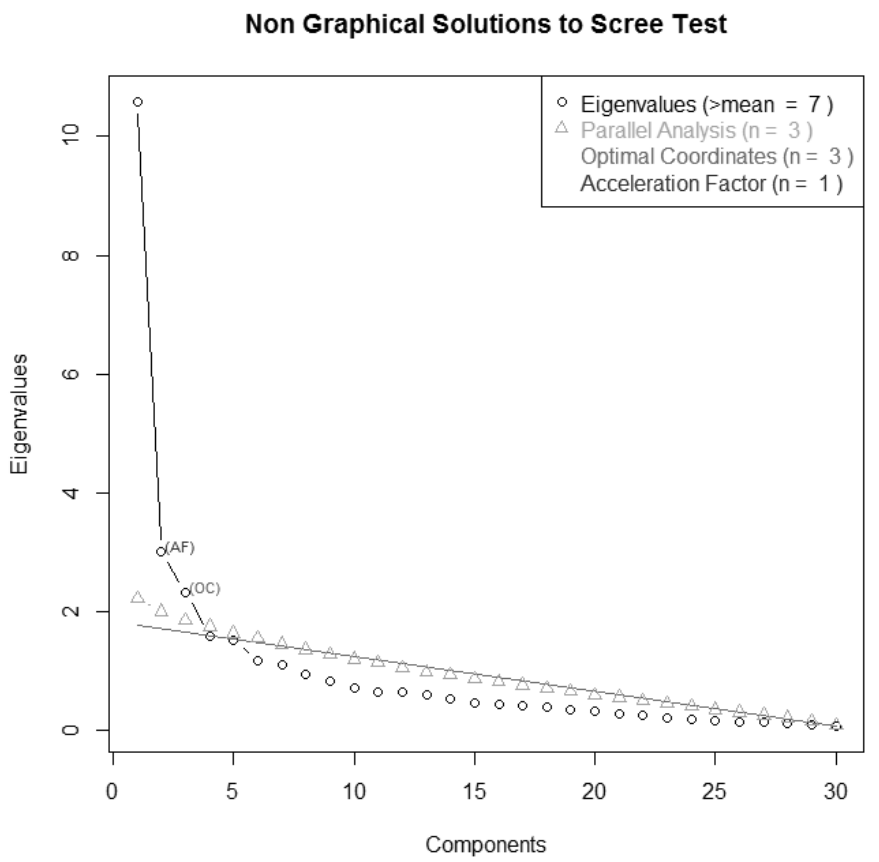

Figura 1. Soluções não gráficas da Escala de Competências Emocionais. confiabilidade. 
Apesar do presente artigo incluir uma análise em TRI, escolheu-se reportar o coeficiente alfa de Cronbach em função de seu frequente uso na literatura. $\mathrm{O}$ coeficiente alfa obtido antes da remoção de itens foi de 0,97, com apenas Q1, Q3 e Q70 apresentando correlação item-total inferior a 0,30 . O coeficiente de confiabilidade empírica calculado pelo pacote mirt também apresentou o valor de 0,97 .
Após a remoção de itens, no processo de ajuste à TRI, o coeficiente alfa foi reduzido para 0,93 e nenhum dos itens apresentou correlação item-total inferior a 0,30 . Por sua vez, o coeficiente de confiabilidade empírica apresentou o valor de 0,94. Na Figura 2, é possível observar que o instrumento permanece com valores de confiabilidade elevada para a maior porção do continuum de traço latente.

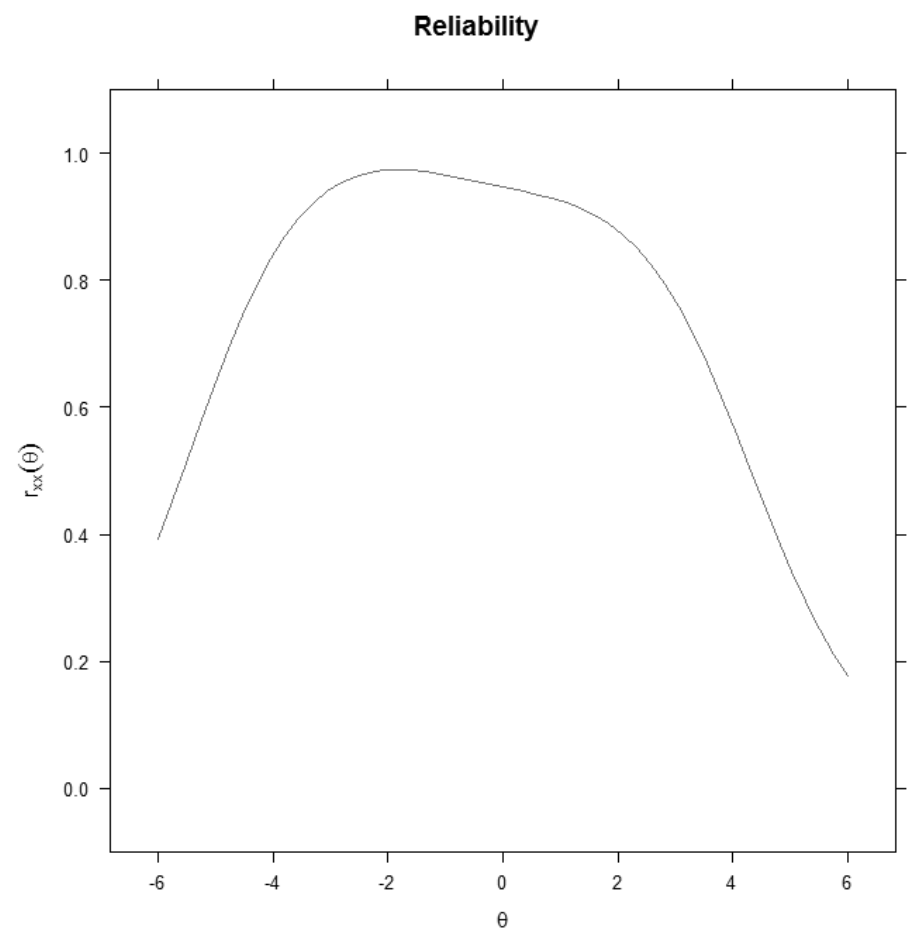

Figura 2. Gráfico de confiabilidade para a ECE apresentando intervalo de níveis de habilidade entre -6 e +6 .

\section{Ajuste dos Itens e Localização}

O primeiro parâmetro localizado para a seleção de itens foi o teste do chi-quadrado, com o objetivo de eliminar itens que tinham uma aproximação empobrecida entre a curva de característica do item e os padrões de resposta observados. Nesse sentido, os itens problemáticos foram os itens Q11 ( $\left.X^{2}=19,66, p=0,033\right), \mathrm{Q} 12$ $\left(X^{2}=20,22, p=0,042\right), \mathrm{Q} 17\left(X^{2}=21, p=0,021\right)$, Q29 $\left(X^{2}=25,73, p=0,012\right)$, Q39 $\left(X^{2}=20,94\right.$, $p=0,022), \mathrm{Q} 50\left(X^{2}=19,65, p=0,033\right), \mathrm{Q} 55\left(X^{2}\right.$ $=25,67, p=0,042)$ e Q64 $\left(X^{2}=23,89, p=0,012\right)$.

O segundo passo foi a exclusão de itens que apresentavam nível de discriminação inferior a 0,70 e superiores a 1,50 . Os itens removidos por nível de discriminação baixo foram os seguintes: Q1 $(\mathrm{a}=0,68), \mathrm{Q} 3(\mathrm{a}=0,41), \mathrm{Q} 6(\mathrm{a}=0,31)$, Q25 ( $\mathrm{a}=0,57), \mathrm{Q} 40(\mathrm{a}=0,61), \mathrm{Q} 55(\mathrm{a}=0,38), \mathrm{Q} 61$ $(\mathrm{a}=0,36), \mathrm{Q} 70(\mathrm{a}=0,25)$ e $\mathrm{Q} 71(\mathrm{a}=0,57)$. É válido apontar que todos os itens mencionados com baixa discriminação apresentaram funções de informação do item representadas praticamente por linhas retas ou de baixo relevo. A quantidade de itens, por outro lado, removidos por excesso de discriminação foi elevada, incluindo os itens Q2 $(a=1,56), \mathrm{Q} 11(a=2,03), \mathrm{Q} 20(a=1,55)$, Q24 $(a=1,84), \mathrm{Q} 26(a=1,93), \mathrm{Q} 27 \quad(a=3,19)$, Q28 $(a=1,96), \mathrm{Q} 30(a=3,25), \mathrm{Q} 35(a=2,05)$, Q36 $(a=2,73), \mathrm{Q} 37(a=3,07), \mathrm{Q} 38(a=3,37)$, Q41 $(a=2,22), \mathrm{Q} 43(a=2,42), \mathrm{Q} 44(a=1,95)$, 
Q47 $(a=2,38), \mathrm{Q} 48(a=1,67), \mathrm{Q} 50(a=1,50)$, Q51 $(a=2,27)$, Q53 $(a=1,88)$, Q54 $(a=1,75)$, Q57 $(a=1,67)$, Q59 $(a=1,83)$, Q63 $(a=2,28)$,
Q64 ( $a=1,56), \mathrm{Q} 65(a=1,65), \mathrm{Q} 66(\mathrm{a}=2,15), \mathrm{Q} 68$ $(a=1,63)$, Q69 $(a=1,79)$ e Q73 $(a=1,97)$. Parâmetros e ajuste dos itens remanescentes estão listados na Tabela 1 .

Tabela 1

Parâmetros de Discriminação (a), Localização (b) e Ajuste dos Itens pelo Teste do Chi-Quadrado incluindo Estatística do Teste $\left(\mathrm{X}^{2}\right)$, Graus de Liberdade (gl) e Nível de Significância Estatística (p-sig)

\begin{tabular}{|c|c|c|c|c|c|}
\hline Lista de itens & $a$ & $b$ & $X^{2}$ & $g l$ & p-sig \\
\hline Expressão Emocional & - & - & - & - & - \\
\hline Q4 & 1.06 & -1.70 & 16.95 & 12 & 0.152 \\
\hline Q8 & 1.07 & -1.08 & 20.13 & 13 & 0.092 \\
\hline Q10 & 1.14 & -1.69 & 10.60 & 11 & 0.478 \\
\hline Q15 & 1.29 & -0.47 & 12.14 & 11 & 0.353 \\
\hline Q18 & 1.29 & -0.44 & 17.64 & 14 & 0.224 \\
\hline Q19 & 1.30 & -1.07 & 7.75 & 10 & 0.654 \\
\hline Q21 & 1.42 & -0.88 & 17.96 & 13 & 0.159 \\
\hline Q33 & 1.05 & -1.26 & 12.18 & 10 & 0.273 \\
\hline Q34 & 1.23 & -0.89 & 13.59 & 11 & 0.257 \\
\hline Q42 & 1.44 & -0.75 & 9.58 & 11 & 0.568 \\
\hline Q46 & 1.19 & -0.76 & 17.10 & 12 & 0.146 \\
\hline Q56 & 0.94 & -0.99 & 18.78 & 12 & 0.094 \\
\hline Q60 & 1.30 & -0.86 & 15.01 & 11 & 0.182 \\
\hline Q62 & 1.32 & -0.97 & 14.48 & 11 & 0.208 \\
\hline Q72 & 1.13 & -0.70 & 10.37 & 12 & 0.584 \\
\hline Compreensão emocional & - & - & - & - & - \\
\hline Q5 & 1.10 & -1.13 & 11.24 & 15 & 0.736 \\
\hline Q7 & 1.14 & -0.61 & 16.74 & 12 & 0.160 \\
\hline Q9 & 1.19 & -0.87 & 11.63 & 11 & 0.392 \\
\hline Q13 & 0.82 & -0.69 & 13.63 & 12 & 0.325 \\
\hline Q16 & 0.75 & -0.89 & 10.68 & 12 & 0.556 \\
\hline Q23 & 1.43 & -0.81 & 14.36 & 12 & 0.278 \\
\hline Q31 & 1.22 & -1.25 & 6.04 & 10 & 0.812 \\
\hline Q45 & 1.32 & -0.67 & 13.09 & 12 & 0.362 \\
\hline Q52 & 1.19 & -0.63 & 10.11 & 12 & 0.606 \\
\hline Q58 & 1.47 & -0.67 & 11.17 & 10 & 0.344 \\
\hline Regulação Emocional & - & - & - & - & - \\
\hline Q14 & 0.75 & -1.30 & 17.05 & 14 & 0.253 \\
\hline Q22 & 1.09 & -1.67 & 13.77 & 12 & 0.316 \\
\hline Q32 & 1.16 & -1.43 & 7.41 & 12 & 0.830 \\
\hline Q49 & 1.22 & -1.40 & 8.68 & 11 & 0.652 \\
\hline Q67 & 0.85 & -0.84 & 16.32 & 13 & 0.232 \\
\hline
\end{tabular}


Considerando os itens que apresentaram o menor nível de localização ou menos reveladores, pode-se listar Q4 $(b=-1,70), \mathrm{Q} 10(b=-1,69)$, Q22 $(b=-1,67)$, Q32 $(b=-1,43)$ e Q49 $(b=-1,40)$. Por sua vez, os itens mais reveladores ou que exigiram o maior nível de traço foram: Q18 $(b=-0,44)$, Q15 $(b=-0,47)$, Q7 $(b=-0,61)$, Q52 $(b=-0,63)$ e Q58 $(b=-0,67)$. Já os itens que apresentaram os níveis mais elevados de discriminação foram: Q45 $(a=1,32), \mathrm{Q} 21(a=1,42), \mathrm{Q} 23$ $(a=1,43), \mathrm{Q} 42(a=1,44)$ e Q58 $(a=1,47)$.
Para os itens remanescentes, a Função de Informação do teste disponível na Figura 3 indica que o erro de medida cresce de forma acelerada especialmente antes da região próxima de níveis de traço localizados em $b=-4,00$ e próximos a $+2,00$. O pico de informação também antecede de forma marcante a localização de $b=0,00$. A informação é essencial para compreender qual seria a melhor forma de uso da escala. Nesse sentido, instrumentos com nível de informação maior em níveis baixos de traço são mais úteis enquanto instrumentos de rastreio.

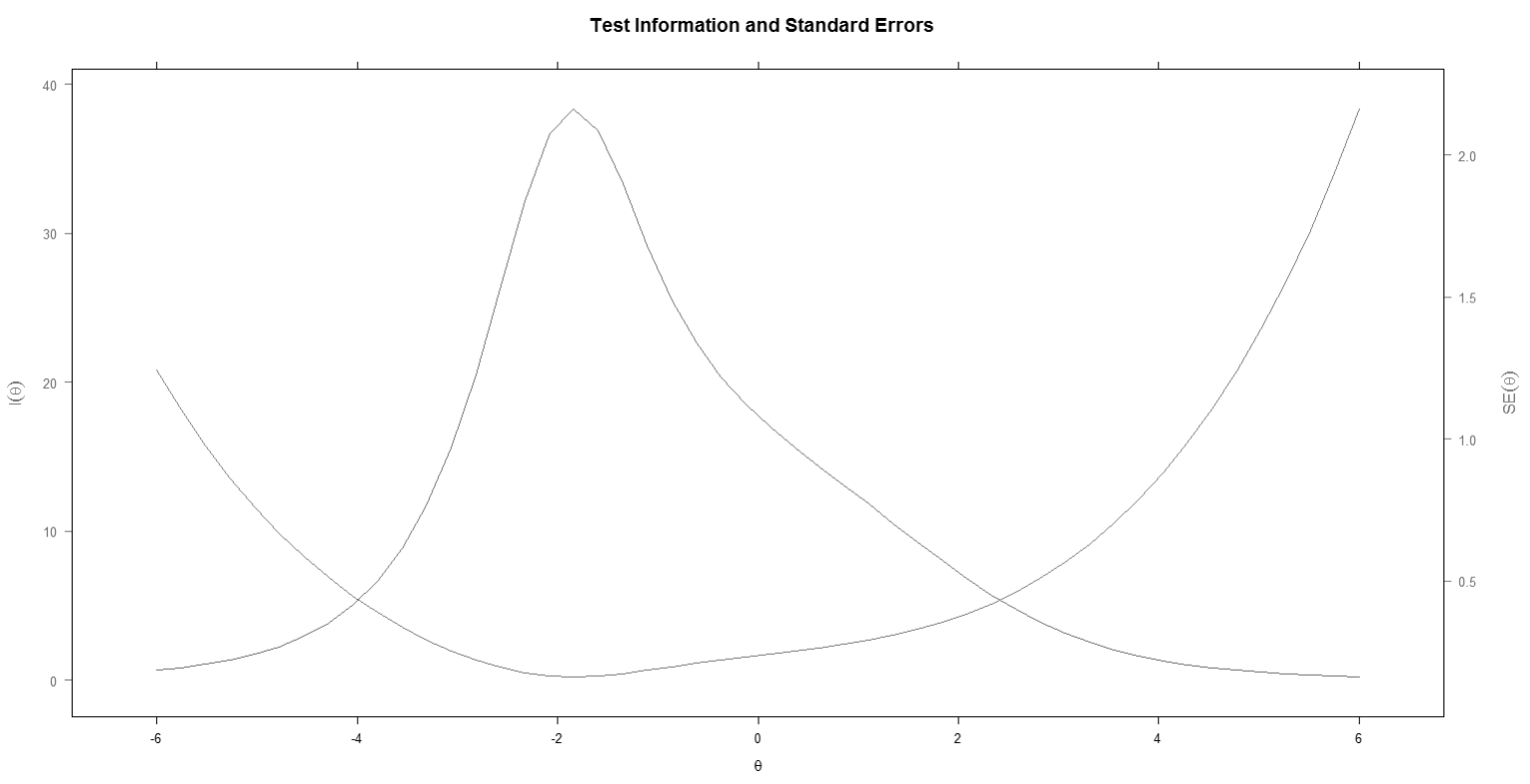

Figura 3. Função de informação do teste. A curva em cor azul indica a curva de informação, enquanto que a curva em tom rosa indica a distribuição do erro.

\section{Discussão e Considerações Finais}

A COMPE foi construída a partir de três fases, a saber: (1) elaboração de itens a partir do polo teórico, sendo estabelecidos 104 itens; (2) avaliação destes itens por juízes, sendo retidos apenas aqueles que atingiram um coeficiente de validade de conteúdo satisfatório, levando a um novo total de 73 itens, (3) submissão da versão com 73 itens a procedimentos de análise de itens através da Teoria de Resposta ao Item, sendo retidos 30 itens no total, finalizando um percurso rigoroso para uma primeira versão com potencial de uso.
Os resultados das análises são indicativos de que se obteve uma primeira versão da COMPE com potencial de uso. Isto configura uma contribuição metodológica a pesquisas na área de estudos em que se insere, na medida em que instrumentos brasileiros que ofereçam uma medida confiável e válida sobre crenças parentais relativas a competências emocionais em crianças não foram identificados. Entende-se que o que pais pensam e acreditam a respeito de como a criança se desenvolve emocionalmente e de quais capacidades principais nesse domínio deve desenvolver, é um ponto crítico e impactante para o desenvolvimento pleno e saudável (Denham, 2007; Parker et al., 2012; Trommsdorff \& Cole, 
2011). Pode-se afirmar que no presente estudo foi dado um passo inicial na constituição de um instrumento que reflita esta concepção teórica, de forma que a COMPE em sua forma atingida oferece índices de confiabilidade e de validade satisfatórios.

Através dos resultados foi possível chegar a um conjunto de itens cuja curva do modelo se ajusta às respostas observadas. Por outro lado, a representação dos comportamentos e a capacidade de discriminação seguem como um desafio para as próximas versões. A primeira questão refere-se à distribuição dos níveis de dificuldade, que apresentaram uma característica de localização predominantemente negativa. Significa que a maior parte dos traços elencados exige uma atitude relativamente baixa referente à atitude alvo. Um dos objetivos futuros para novas versões consistirá na inclusão de itens que representem atitudes menos disponíveis ou de maior grau de dificuldade. Da mesma forma, tais itens tornariam possível uma maior sensibilidade em níveis de traço mais elevados do que o exibido por exemplo na Figura 3 no presente artigo.

Sobre os níveis de dificuldade, um dos pontos de atenção foi o de que os itens Q18, Q15, Q7, Q52 e Q58 atingiram os maiores níveis de dificuldade. Talvez isso esteja relacionado ao fato de quase todos, à exceção do Q58, estarem associados a emoções consideradas negativas (como a culpa, a vergonha e a raiva). Tal resultado pode, portanto, estar associado a um traço cultural das crenças parentais (Denham, 2007; Trommsdorff \& Cole, 2011) não sendo muito esperado e valorizado pelos pais brasileiros que crianças na faixa de idade contemplada tenham capacidades vinculadas à sua expressão ou compreensão.

É possível afirmar que uma das vantagens do presente estudo consiste na elaboração de uma medida para crenças parentais sobre competências emocionais que considera $o$ contexto de desenvolvimento da criança, no caso, o de pais e mães brasileiros. A segunda vantagem do presente estudo consiste no uso da Teoria de Resposta ao Item como procedimento de análise. Dentre as vantagens listadas por Hambleton, Swaminathan e Rogers (1991) é possível destacar: (a) uma caracterização mais adequada da relação entre medida e o erro de medida; (b) melhor caracterização de informações relacionadas à escalabilidade de itens e pessoas, assim como uma informação que contribui de forma rica para a seleção de itens e, por fim; (c) estimativas que normalmente não são amostra ou teste-dependente.

Dentre as fragilidades do estudo podemos apontar a amostra limitada. O objetivo de restringir a faixa etária na qual deveriam estar inseridos os filhos dos pais entrevistados foi o de propor um instrumento adequado a um contexto real e que tivesse aderência ao indicado pela autora do modelo teórico em sua experiência prática com crianças na idade em que o desenvolvimento dessas competências ganha maior destaque (Denham, 2007). Entendeu-se que as noções e cognições de pais e mães sobre as competências emocionais de crianças é algo que tanto está situado em um contexto sociocultural específico, quanto sofre mudanças no decorrer das etapas do ciclo vital dos filhos.

Outro motivo que se pode hipotetizar para a restrição do tamanho da amostra é a dificuldade na obtenção de participantes que se dispusessem a ocupar uma parcela de cerca de 20 a 30 minutos de seu tempo para preencher o formulário online. Os participantes pretendidos, em boa parte, eram pessoas que se encontravam em um momento da vida em que se presume exercerem uma atividade profissional (estarem trabalhando) e na condição de pais de uma criança, com toda a responsabilidade e afazeres que isso implica. Tentou-se contornar o tamanho da amostra usando técnicas de reamostragem para oferecer maior robustez à estimativa e aos testes de ajuste.

Pode-se pensar ainda, em termos de limitações do estudo, que não foi explorado o nível de conhecimento dos pais acerca das suas próprias competências emocionais, o que pode vir a impactar os resultados obtidos e permitir uma melhor interpretação dos mesmos. Pontua-se a conveniência de tal verificação em investigações futuras. Por outro lado, não há dúvida de que as análises aqui expostas necessitam no futuro de ampliação do poder estatístico. 
Para além do poder estatístico, outra expectativa futura também consiste na realização de testes sobre a estabilidade das crenças sobre competências emocionais relativas às idades estudadas, com o objetivo de compreender como elas podem se modificar ao longo do tempo. Tomando-se por base a concepção de que crenças parentais são constituídas de acordo com modelos culturais específicos e significados compartilhados pelos membros do contexto sociocultural em que se vive, entende-se que passem por mudanças no decorrer do tempo histórico.

Ponderando limitações e contribuições do estudo, entende-se que foi atingido seu propósito de expor as etapas de construção da escala COMPE e verificar os parâmetros psicométricos preliminares como dimensionalidade e confiabilidade dos itens. Os resultados obtidos com a aplicação realizada são consistentes com o modelo empregado. Considera-se que a escala apresenta propriedades que permitem sua utilização em pesquisas envolvendo crenças parentais e emoções, com confiabilidade. Ademais, trata-se de instrumento construído especialmente para uso com população brasileira, o que representa um avanço tendo em vista a falta de instrumentos com esse foco e objetivo no país.

\section{Referências}

Baker, F. (2001). The Basics of Item Response Theory. Washington: ERIC Clearinghouse on Assessment and Evaluation.

Bohanek, J., Fivush, R., Zaman, W., Lepore, C., Merchant, S., \& Duke, M. (2009). Narrative interaction in family dinnertime conversations. Merrill-Palmer Quarterly, 55(4), 488-515. doi: 10.1353/mpq.0.0031

Boyatzis, R. E., \& Sala, F. (2004). Assessing emotional intelligence competencies. In G. Geher (Ed.), The measurement of emotional intelligence (pp. 147-80). New York: Novas Science.

Chalmers, R. P. (2012). mirt: A multidimensional item response theory package for the $\mathrm{R}$ environment. Journal of Statistical Software, 48(6), 1-29. doi: http://hdl.handle.net/10.18637/jss v048.i06
Curby, T. W., Brown, C. A., Bassett, H. H., \& Denham, S. A. (2015). Associations between preschoolers' social-emotional competence and preliteracy skills. Infant and Child Development, 24, 549-570. doi: https://doi.org/10.1002/ icd.1899

Damásio, A. R. (2009). Em busca de Espinosa: Prazer e dor na ciência dos sentimentos. São Paulo, SP: Companhia das Letras.

De Ayala, R. J. (2009). The Theory and practice of item Response Theory. New York: The Guilford Press.

Denham, S. A. (1998). Emotional development in young children. New York: The Guilford Press.

Denham, S. A. (2007). Dealing with feelings: How children negotiate the worlds of emotions and social relationships. Cognition, Brain \& Behavior, 11, 1-48.

Denham, S. A., Bassett, H. H., Zinsser, K., \& Wyatt, T. M. (2014). How preschoolers' social-emotional learning predicts their early school success: Developing theory-promoting, competency-based assessments. Infant and Child Development, 23, 426-454. doi: https://doi.org/10.1002/ icd. 1840

Denham, S. A., Ferrier, D. E., Howarth, G. Z., Herndon, K. J., \& Bassett, H. H. (2016). Key considerations in assessing young children's emotional competence. Cambridge Journal of Education, 46(3), 299-317. doi: https://doi.org/10.1080/030 5764X.2016.1146659

Finlon, K. J., Izard, C. E., Seidenfeld, A., Johnson, S. R., Cavadel, E. W., Ewing, E. S. K., \& Morgan, J. K. (2015). Emotion-based preventive intervention: Effectively promoting emotion knowledge and adaptive behavior among at-risk preschoolers. Development and Psychopathology, 27, 1353-1365. doi: 10.1017/ S0954579414001461

Fivush, R. (2008). Remembering and reminiscing: How individual lives are constructed in family narratives. Memory Studies, 1, 49-58. doi: https://doi.org/10.1177/1750698007083888

Goleman, D. (1995). Inteligência Emocional - A teoria revolucionária que redefine o que é ser inteligente. Rio de Janeiro, RJ: Objetiva.

Gottman, J. M., Katz, L. F., \& Hooven, C. (1996). Parental Meta-Emotion Philosophy and the emotional life of families: Theoretical models and 
preliminary data. Journal of Family Psychology, 10(3), 243-268.

Halberstadt, A. G., Dunsmore, J. C., Bryant, A., Jr., Parker, A. E., Beale, K. R., \& Thompson, J. A. (2013). Development of the Parents' Beliefs about Children's Emotions Questionnaire. Psychological Assessment, 25, 1195-1210. doi: 10.1037/a0033695

Hambleton, R. K., Swaminathan, H., \& Rogers, H. J. (1991). Fundamentals of item response theory. London: Sage.

Harkness, S., \& Super, C. (2010). Parental ethnotheories in children learning. In D. F. Lancy, J. Bock, \& S. Gaskins (Eds.), The Anthropology of Learning in childhood (pp. 65-84). Plymouth, UK: AltaMira Press.

Izard, C., Fine, S., Schultz, D., Mostow, A., Ackerman, B., \& Youngstrom, E. (2001). Emotional knowledge as a predictor of social behavior and academic competence in children at risk. Psychological Science, 12, 18-23. doi: https:// doi.org/10.1111/1467-9280.00304

Lozada, F. T., Halberstadt, A. G., Craig, A. B., Dennis, P. A., \& Dunsmore, J. C. (2016). Parents' beliefs about children's emotions and parents' emotion-related conversations with their children. Journal of Child and Family Studies, 25, 1525-1538.

Mendonça, S. S. (2009). Competências profissionais dos enfermeiros: A excelência do cuidar. Penafiel, Portugal: Penagráfica-Artes.

Parker, A. E, Halberstadt, A. G., Dunsmore, J. C., Townley, G., Bryant, A., Thompson, J. A., \& Beale, K. S. (2012). Emotions are a window into one's heart": A qualitative analysis of parental beliefs about children's emotions across three ethnic groups. Monographs of the Society for Research in Child Development, 77(3), 1-136. doi: $10.1111 / \mathrm{j} .1540-5834.2012 .00676 . x$
Pasquali, L. (1998). Psicometria: Teoria e aplicações. Brasília, DF: Editora da Universidade de Brasília.

Pasquali, L. (2010a). Instrumentação Psicológica: Fundamentos e Práticas. Porto Alegre, RS: Artmed.

Pasquali, L. (2010b). Testes referentes a construto: Teoria e modelo de construção. In L. Pasquali (Ed.), Instrumentação psicológica: Fundamentos e práticas (pp. 165-198). Porto Alegre, RS: Artmed.

Pasquali, L. (2011). Psicometria: Teoria dos testes na psicologia e na educação. Petrópolis, RJ: Vozes.

Raiche, G., \& Magis, D. (2010). nFactors: An R package for parallel analysis and non graphical solutions to the Cattell scree test ( $\mathrm{R}$ package version 2.3). Montreal.

Saarni, C. (1999). How we become emotionally competent. In C. Saarni (Ed.), The Development of Emotional Competence (pp. 54-78). New York: The Guilford Press.

Sheffield-Morris, A., Silk, J., Steinberg, L., Myers, S., \& Robinson, L. (2007). The role of the family context in the development of emotion regulation. Social Development, 16, 361-388. doi: 10.1111/j.1467-9507.2007.00389.x

Trommsdorff, G., \& Cole, P. M. (2011). Emotion self-regulation, and social behavior in cultural contexts. In X. Chen \& K. H. Rubin (Eds.), Socioemotional development in cultural context (pp. 131-163). New York: The Guilford Press.

Recebido: 29/12//2016

$1^{a}$ revisão: $14 / 08 / 2017$ Aceite final: 06/11/2017 


\section{Anexo}

\section{COMPE - Relação de itens do Formulário Eletrônico}

O quanto você concorda que "É importante para o desenvolvimento da criança que ela..."

\begin{tabular}{|c|c|c|}
\hline Original* & Final** & Itens \\
\hline Q4 & Q1 & Expresse a emoção que sente quando está contente brincando com outras crianças. \\
\hline Q5 & Q2 & Entenda que o que outra pessoa (adulto ou criança) está sentindo se chama medo. \\
\hline Q7 & Q3 & $\begin{array}{l}\text { Saiba que se alguém está se mostrando com raiva é porque ocorreu algo que lhe irritou ou abor- } \\
\text { receu muito. }\end{array}$ \\
\hline Q8 & Q4 & Expresse a emoção que sente quando outra criança faz alguma coisa que estraga a brincadeira. \\
\hline Q9 & Q5 & Compreenda que o que outra pessoa (adulto ou criança) está sentindo é tristeza. \\
\hline Q10 & Q6 & Demonstre alegria quando está feliz. \\
\hline Q13 & Q7 & Saiba que o que outra pessoa (adulto ou criança) está sentindo se chama orgulho. \\
\hline Q14 & Q8 & $\begin{array}{l}\text { Peça ajuda à mãe ou outra pessoa em quem confie, quando está com muita raiva porque uma crian- } \\
\text { ça tomou o seu brinquedo e quebrou. }\end{array}$ \\
\hline Q15 & Q9 & Mostre que ficou com raiva quando alguma coisa que queria não deu certo. \\
\hline Q16 & Q10 & $\begin{array}{l}\text { Compreenda que se alguém parece envergonhado é porque está sem jeito diante de outras pessoas } \\
\text { ou de certas situações. }\end{array}$ \\
\hline Q18 & Q11 & Expresse vergonha quando se sente constrangida diante de alguém ou por alguma situação. \\
\hline Q19 & Q12 & Expresse a emoção que sente quando vê um cão latindo se aproximando de forma ameaçadora. \\
\hline Q21 & Q13 & Manifeste orgulho quando fez algo que sabe que seus pais consideram muito importante. \\
\hline Q22 & Q14 & Encontre uma maneira eficiente de buscar ajuda quando precisa suportar o medo que está sentindo. \\
\hline Q23 & Q15 & Compreenda que o que outra pessoa (adulto ou criança) está sentindo se chama raiva. \\
\hline Q31 & Q16 & Entenda que o que está sentindo naquela situação é tristeza e não raiva. \\
\hline Q32 & Q17 & $\begin{array}{l}\text { Peça ajuda à mãe ou outra pessoa em quem confie, para conseguir aguentar o medo e não entrar } \\
\text { em pânico. }\end{array}$ \\
\hline Q33 & Q18 & Demonstre que está orgulhosa por ter conseguido fazer aquilo que tanto queria. \\
\hline Q34 & Q19 & Expresse que está brava quando alguma coisa que não lhe agrada acontece. \\
\hline Q42 & Q20 & Expresse a emoção que sente quando seu brinquedo favorito cai no chão e quebra todo. \\
\hline Q45 & Q21 & Compreenda que o que outra pessoa (adulto ou criança) está sentindo é vergonha. \\
\hline Q46 & Q22 & Mostre que ficou com raiva quando aconteceu algo que lhe contrariou. \\
\hline Q49 & Q23 & $\begin{array}{l}\text { Controle a raiva para não agredir outra criança que tomou o seu brinquedo e quebrou, mesmo que } \\
\text { fique muito aborrecida. }\end{array}$ \\
\hline Q52 & Q24 & Tenha conhecimento de que o que outra pessoa (adulto ou criança) está sentindo é culpa. \\
\hline Q56 & Q25 & Demonstre que ficou com raiva quando outra criança lhe bateu com força. \\
\hline Q58 & Q26 & Entenda que se alguém está expressando alegria significa que algo de bom aconteceu. \\
\hline Q60 & Q27 & Demonstre medo quando vê um cão latindo se aproximando de forma ameaçadora. \\
\hline Q62 & Q28 & Demonstre orgulho quando fez algo que agradou muito a seus pais. \\
\hline Q67 & Q29 & Regule a forma como mostra suas emoções para que sejam adequadas à situação e ao ambiente. \\
\hline Q72 & Q30 & Demonstre que está com medo quando ouve algo que a deixa assustada. \\
\hline
\end{tabular}

Obs: *Numeração original referente à versão inicial, anterior à eliminação de itens nos procedimentos de TRI. **Numeração final refere-se ao número do item após a exclusão. 